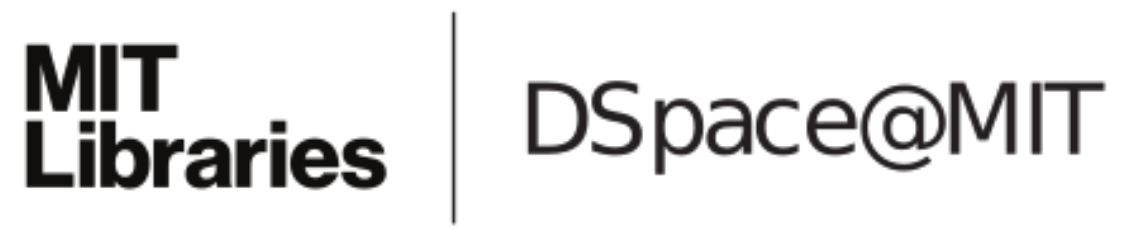

\author{
MIT Open Access Articles
}

Deionization shock driven by electroconvection in a circular channel

The MIT Faculty has made this article openly available. Please share how this access benefits you. Your story matters.

As Published: 10.1103/PHYSREVFLUIDS.4.113701

Publisher: American Physical Society (APS)

Persistent URL: https://hdl.handle.net/1721.1/136527

Version: Final published version: final published article, as it appeared in a journal, conference proceedings, or other formally published context

Terms of Use: Article is made available in accordance with the publisher's policy and may be subject to US copyright law. Please refer to the publisher's site for terms of use. 


\title{
Deionization shock driven by electroconvection in a circular channel
}

\author{
Zhibo Gu, ${ }^{1}$ Bingrui Xu, ${ }^{1}$ Peng Huo, ${ }^{1}$ Shmuel M. Rubinstein, ${ }^{2}$ \\ Martin Z. Bazant, ${ }^{3}$ and Daosheng Deng $\oplus^{1, *}$ \\ ${ }^{1}$ Department of Aeronautics and Astronautics, Fudan University, Shanghai 200433, China \\ ${ }^{2} J o h n$ A. Paulson School of Engineering and Applied Sciences, Harvard University, Cambridge, \\ Massachusetts 02138, USA \\ ${ }^{3}$ Department of Chemical Engineering and Department of Mathematics, Massachusetts Institute of \\ Technology, Cambridge, Massachusetts 02139, USA
}

(Received 29 January 2019; published 5 November 2019)

\begin{abstract}
In a circular channel passing overlimiting current (faster than diffusion), transient vortices of bulk electroconvection are observed in a salt-depleted region within the horizontal plane. The spatiotemporal evolution of the salt concentration is directly visualized, revealing the propagation of a deionization shock wave driven by bulk electroconvection up to millimeter scales. This mechanism leads to quantitatively similar dynamics as for deionization shocks in charged porous media, which are driven instead by surface conduction and electro-osmotic flow at micron to nanometer scales. The remarkable generality of deionization shocks under overlimiting current could be used to manipulate ion transport in complex geometries for desalination and water treatment.
\end{abstract}

DOI: 10.1103/PhysRevFluids.4.113701

\section{INTRODUCTION}

Ion transport in electrochemical cells is essential for electrochemical energy storage, desalination for water treatment, and biomedical applications [1-3]. Designing complex geometries is one of the typical approaches to control ion transport, as illustrated by the stack of alternating cation- and anion-exchange membranes in classical electrodialysis (ED) [4]. More recently, ion enrichment/depletion resulting from overlapping electric double layers in micro/nanochannels [5] has been applied to biomolecule separation [6]. Polarizable porous electrodes or particles under applied voltages can also induce capacitive deionization, in a variety of geometries $[7,8]$.

In these and other applications, many intriguing phenomena are associated with the passage of overlimiting current (OLC), faster than diffusion, to an ion-selective membrane [9] or electrode [10,11]. Physical (as opposed to chemical [12]) mechanisms for OLC fall into two general categories: bulk electroconvection (EC) associated with extended space charge on the membrane [13-18] and surface charge (SC) effects, namely surface conduction and electro-osmotic flow (EOF), through charged microchannels or porous media leading to the membrane or electrode [10,11,19-23]. The transient response to OLC can involve the shocklike propagation of a sharp drop in salt concentration [24-26]. The propagation of SC-driven "deionization shocks" (DS) in charged porous media [27] has been exploited for water desalination and purification in the emerging process of "shock electrodialysis" $[20,21,28,29]$ and for control of metal growth in "shock electrodeposition" $[10,11]$. Since EC-driven vortices can also sustain OLC by creating an extended salt depletion zone, it is interesting to explore whether EC alone can give rise to DS.

*dsdeng@fudan.edu.cn 

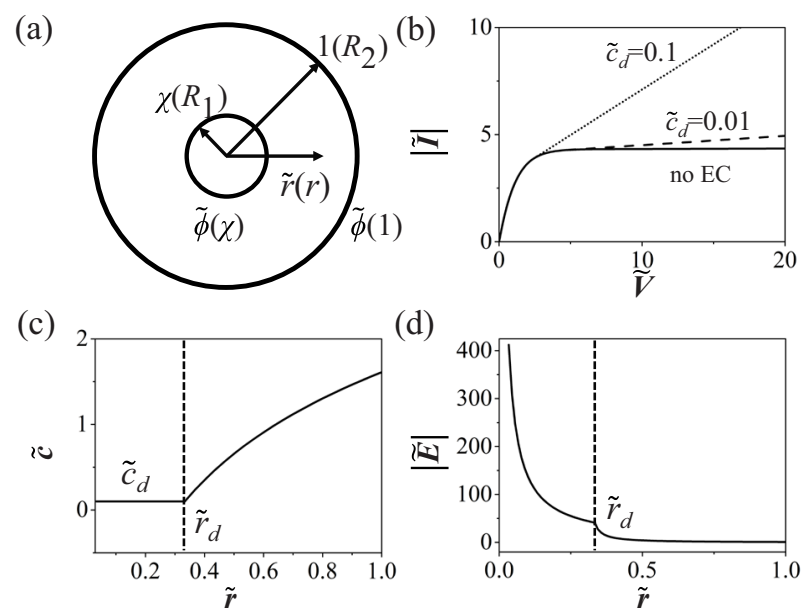

FIG. 1. (a) Sketch of a circular channel. The positive voltage bias for $\tilde{\phi}(1)>\tilde{\phi}(\chi), \tilde{I}<0$. (b) OLC dependent on $\tilde{c}_{d}$, (c) concentration distribution, and (d) profile of the electric field $\left(\tilde{\phi}=35, \tilde{c}_{d}=0.1, R_{d}=\right.$ $\left.\tilde{r}_{d} R_{2}\right)$.

In this paper, we report the observation of EC-driven DS in a circular microchannel. Vortices reminiscent of bulk EC are identified within the horizontal plane, and the spatiotemporal evolution of concentration is directly visualized. Propagation of EC has a remarkable agreement with the proposed model.

\section{SIMPLE MODEL OF EC-DRIVEN OLC}

Consider the following model problem, studied experimentally below. A dilute, binary $z: z$ electrolyte with concentration $\left(c_{0}\right)$ fills a circular channel with an inner radius $\left(R_{1}\right)$ and outer radius $\left(R_{2}\right)\left(\chi=R_{1} / R_{2}<1\right)$ under an applied voltage [Fig. 1(a)]. In the steady state, under the assumption of the azimuthal symmetry and charge neutrality, the Nernst-Planck equations are simplified into a 1D dimensionless form, equating cation flux to current density and anion flux to zero for an ideal cation-selective surface [19]:

$$
\begin{aligned}
& \frac{d \tilde{c}}{d \tilde{r}}+\tilde{c} \frac{d \tilde{\phi}}{d \tilde{r}}+\tilde{\sigma}_{E C} \frac{d \tilde{\phi}}{d \tilde{r}}=-\frac{\tilde{I}}{2 \pi \tilde{r}}, \\
& \frac{d \tilde{c}}{d \tilde{r}}-\tilde{c} \frac{d \tilde{\phi}}{d \tilde{r}}+\tilde{\sigma}_{E C} \frac{d \tilde{\phi}}{d \tilde{r}}=0,
\end{aligned}
$$

where $\tilde{c}=\tilde{c}_{+}=\tilde{c}_{-}$is the (equal) dimensionless mean concentration of cations and anions scaled by $c_{0}, \tilde{r}$ the dimensionless radius scaled by $R_{2}, \tilde{\phi}$ the dimensionless potential scaled by the thermal voltage, $k_{B} T / z e$, and $\tilde{I}$ is the dimensionless current scaled by $z e D c_{0}$, assuming equal diffusivity $D$ for cations and anions.

In contrast to the leaky membrane model $[19,20,23,30]$, where the residual surface conductivity sustaining OLC is provided by excess counterions in a charged porous medium, we have introduced a residual bulk conductivity due to electroconvection $\tilde{\sigma}_{E C}\left(\sigma_{E C} / c_{0}\right)$, which depends on the local electric field $E$ and becomes dominant above a critical threshold $E_{c}$ that is only reached in the depleted zone during OLC, e.g., via $\sigma_{E C}(E, c)=c_{d}(c) \tanh ^{2}\left(E / E_{c}\right)$. Similar to EOF [19,21,22], EC with the intense vortices near the dead end causes the formation of an extended depletion zone $\left(\tilde{r} \leqslant \tilde{r}_{d}\right)$ with a nearly constant area-averaged concentration $\left(\tilde{c}_{d}\right)$ [13-15], resulting in residual conductivity ( $\tilde{\sigma}_{E C} \sim \tilde{c}_{d}$ for both types of ions), while convection is negligible in the bulk region far away from the vortices. 


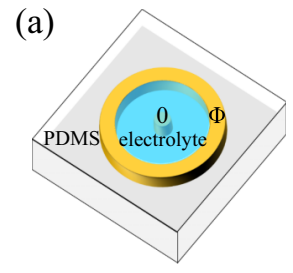

(e)

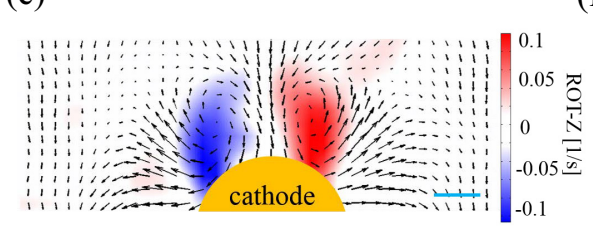

(b)

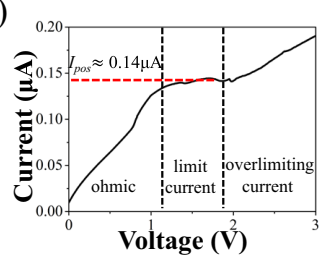

(c)

(f)

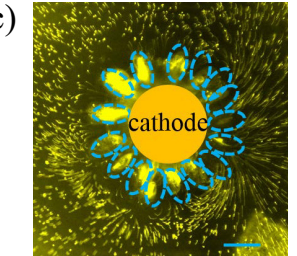

(d)

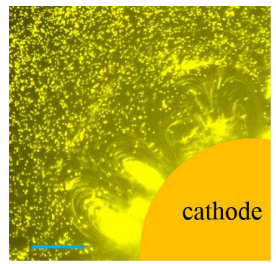

$(\mathrm{g})$

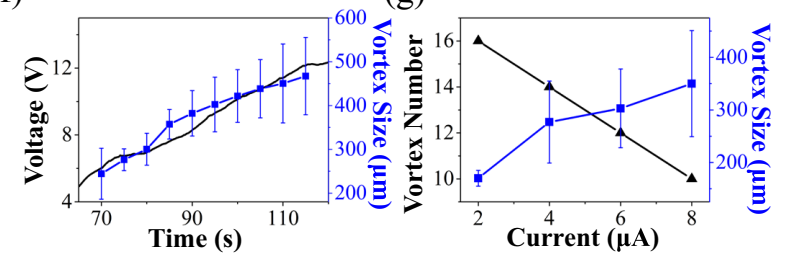

FIG. 2. Vortex observation from a top-down view. (a) Sketch of the PDMS device $\left(2 R_{2}=6 \mathrm{~mm}, H \approx\right.$ $35 \mu \mathrm{m}, \chi=1 / 30$, and $\Phi>0$ for the positive voltage bias). (b) The $I-V$ curve showing $I_{\mathrm{pos}} \approx 0.14 \mu \mathrm{A}$. (c)-(e) Under an applied current at $2 \mu \mathrm{A}$ in the overlimiting current regime, the time-lapse snapshot at $t=60 \mathrm{~s}$ with an exposure time of $5 \mathrm{~s}$ for (c), and with an exposure time of $100 \mathrm{~ms}$ for (d); (e) PIV images with a short exposure time of $40 \mathrm{~ms}$ at $t=20 \mathrm{~s}$ when the first vortex pair occurs. (f) Voltage and vortex size increase with time. $(\mathrm{g})$ The number of vortex decreases with the applied current, while the size of vortex increases with the applied current when the vortex occurs. Scale bar in (c)-(e) for $200 \mu \mathrm{m}$.

This two-region approximation can be used to solve the model, matching the concentration and potential at $\tilde{r}_{d}$, to obtain $I-V$ relationship (as shown in Appendixes A and B),

$$
\tilde{V}=\ln \left(1+\frac{\tilde{I}}{4 \pi \tilde{c}_{d}} \ln \tilde{r}_{d}\right)-\frac{\tilde{I}}{4 \pi \tilde{c}_{d}} \ln \left(\frac{R_{d}}{R_{1}}\right),
$$

where $\tilde{r}_{d}$ can be found for the given $\tilde{I}$ and $\tilde{c}_{d}$ by the concentration conservation. The calculated $I-V$ curves [Fig. 1(b)] demonstrate OLC is sustained by $\tilde{\sigma}_{E C}$. Additionally, concentration profile and electric field are presented in Figs. 1(c) and 1(d), indicating a constant $\tilde{c}_{d}$ and the sharp increase of electric field in the depletion region $\left(\tilde{r} \leqslant \tilde{r}_{d}\right)$ (Appendix B).

\section{CURRENT-VOLTAGE MEASUREMENTS AND VORTEX OBSERVATION}

A PDMS device with a circular channel is illustrated in Fig. 2(a), $2 R_{1}=200 \mu \mathrm{m}$ for the inner copper wire, $2 R_{2}=6 \mathrm{~mm}(\chi=1 / 30)$ for the outer copper ring, and $H \approx 35 \mu \mathrm{m}$ for the channel height. This combination of copper electrodes and $\mathrm{CuSO}_{4}$ solution can avoid complicated chemical reactions to simplify the system [21]. The measured $I-V$ curve [Fig. 2(b)] for $1 \mathrm{mM}$ aqueous $\mathrm{CuSO}_{4}$ solution under the positive voltage bias (a Keithley 2450 Source Meter) is characterized with a linear Ohmic regime, a plateau of limiting current, and OLC. Unlike the straight channel with parallel planar electrodes, $I-V$ curves and limiting currents here depend on the voltage bias (Appendixes A and C). By modifying PDMS surfaces to be positively charged through being immersed into poly(allylamine hydrochloride) (PAH) solution $(1 \mathrm{mg} / \mathrm{ml} \mathrm{PAH} \mathrm{with} 0.1 \mathrm{M} \mathrm{NaCl})$ for $2 \mathrm{~h}$ followed after the air plasma treatment [21], the measured $I-V$ curves were uninfluenced.

As shown in Fig. 2(b), limiting current is subsequently followed by OLC, and the possible mechanism is the formation of vortex to enhance ion transport [15]. In order to visualize vortex, $0.001 \% 1-\mu \mathrm{m}$-diam fluorescent particles (Invitrogen) were added into $1 \mathrm{mM}$ aqueous $\mathrm{CuSO}_{4}$ solution together with $0.1 \%$ Tween 80 (Sigma Aldrich) to avoid particle aggregation [31]. Under an applied current at $2 \mu \mathrm{A}$ the vortices are gradually generated, which are visualized by the fluorescent microscope (Zeiss, Axio Zoom V16). At $t=60 \mathrm{~s}$, the vortex is indicated by the time-lapse snapshot 
[Figs. 2(c) and 2(d)] (see Supplemental Material [32]). By employing particle image velocimetry (PIV), the vortex fields at $t=20 \mathrm{~s}$ are obtained [Fig. 2(e)], revealing a pair of vortices exists near the cathode with velocity up to around $20 \mu \mathrm{m} / \mathrm{s}$.

We simultaneously recorded the increased voltage and built the correlation between vortex size (the vortex length) and electric response [Fig. 2(f)] [31]. By checking the vortices during their occurrence [Fig. 2(g)], vortex size increases with the current while the vortex number decreases with current, since the circumferential length is fixed for a given inner cathode.

Different from the typical bottom-up setup with the vertical concentration gradient or gravitational convection [21,33], here the PDMS device containing the circular channel is placed horizontally and the voltage is applied between the inner wire and outer ring [Fig. 2(a)], and concentration gradient is absent vertically and gravitational convection is irrelevant. In addition, unlike the typical straight microchannels with four sidewalls to cause EOF [19,22], here the gap of this circular channel is only $35 \mu \mathrm{m}$ and EOF due to the bottom and top surface charges might be relevant only along the vertical direction. Again vortices were nearly unaffected by positive-charged PDMS surface, implicating the negligible role of SC mechanism and the essential role of EC.

Hence the observed vortex confined within the horizontal plane with circumferential length up to millimeters is attributed to EC. Similar EC-driven vortices have also observed parallel-plate geometries, in terms of both size $(\sim 100 \mu \mathrm{m})$ and velocity $(\sim 10 \mu \mathrm{m} / \mathrm{s})$ [15,31], and the fastest flow is tangential to the circular cathode surface [Fig. 2(c)]. The vortices are thus consistent with EC instability, although it is beyond our scope to classify the space charge as nonequilibrium [14] or equilibrium [18] or quantify the effect of geometrical curvature.

\section{CONCENTRATION PROFILE EVOLUTION}

The spatiotemporal evolution of the EC-driven DS as visualized through the cation concentration. The concentration of copper ions $\left(\mathrm{Cu}^{2+}\right)$ was detected by the fluorescent indicator, $20 \mu \mathrm{M}$ Phen Green SK dipotassium salt (Invitrogen), the fluorescence intensity of which is quenched by $\mathrm{Cu}^{2+}$ ions, i.e., the increased fluorescence intensity indicates the reduced concentration, and vice versa. $\mathrm{CuSO}_{4}$ electrolyte at $10 \mathrm{mM}$ was prepared in a compound solution (a mixture of the distilled water, thiodiethanol, and dimethyl sulfoxide) to enhance the fluorescent signals. For a constant current at $20 \mu \mathrm{A}$, the typical snapshots of fluorescent signals recorded by the fluorescent microscope (Zeiss, Axio Zoom V16) are shown in Fig. 3(a) (see Supplemental Material [32]).

(a)

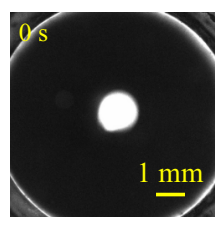

(b)

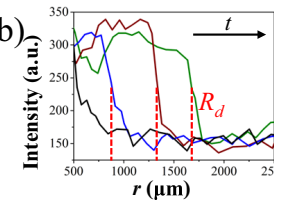

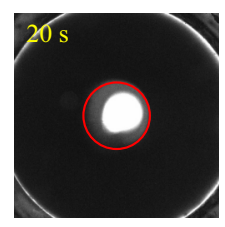

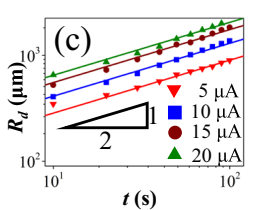

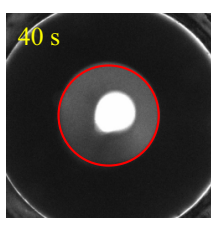

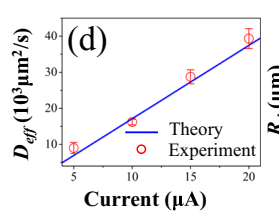

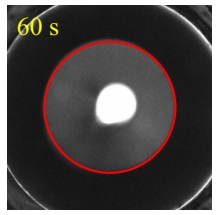

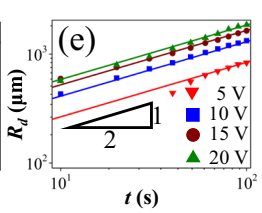

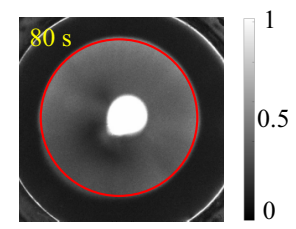

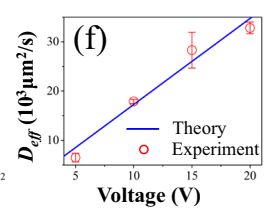

FIG. 3. Spatiotemporal evolution of concentration $\left(R_{1}=100 \mu \mathrm{m}\right)$. (a) Snapshots of fluorescent signals at $20 \mu \mathrm{A}$, red circular contours for the propagation front. Saturated white color in the center due to the extra liquids around the copper wire. (b) Radial profiles of fluorescent intensity at $t=0,20,40$, and $60 \mathrm{~s} ; R_{d}$ is marked by the red dash line at the steep jump of fluorescent intensity due to the concentration depletion. $R_{d}$ has a $1 / 2$ power law under various currents (c) and voltages (e), while $D_{\text {eff }}$ is proportional to the currents (d) and voltages (f) from Eqs. (3) and (4). Error bars in (d) and (f) for the standard deviations from six measurements. 
The initial homogeneous distribution of fluorescent intensity was separated into two distinct regions marked by the red circular contours [Fig. 3(a)], and the radial intensity is quantified in Fig. 3(b), clearly demonstrating the propagation front with a sharp intensity jump. This enhanced intensity was observed only far above the limiting current, but disappeared below the limiting current. In addition, either concentration polarization of the fluorescent indicator or electromigration of the negative-charged Phen Green SK can only cause the decreased fluorescent intensity near the inner cathode. Hence the stronger fluorescent intensity of the inner brighter region demonstrates the significant reduction of copper concentration, reminiscent of previous observations of SC-driven DS in the same electrolyte $[10,11,22]$. In this case, however, SC can be ruled out in favor of EC, since the concentration evolution was found to be nearly identical with a positively charged surface.

\section{SCALING ANALYSIS}

Propagation front $\left(R_{d}\right)$ is located at the middle point of the abrupt jump, as indicated by the dash red lines [Fig. 3(b)]. For various constant currents, $R_{d}$ is fitted by a $1 / 2$ power-law scaling [Fig. 3(c)]. The extracted effective diffusion coefficient $\left(D_{\text {eff }}\right)$ [Fig. 3(d)], unlike the normal diffusion growth of depletion layer prior to the onset of EC [16], is about one order of magnitude higher than the typical diffusion coefficient of copper ions $\left(0.8 \times 10^{3} \mu \mathrm{m}^{2} / \mathrm{s}\right)$.

Physically, under the constant current $(I)$, the current in the depleted region is mainly determined by the electromigration and convection due to vortices; then ion conservation at the shock front implies $I /\left(2 \pi R_{d}\right) \sim d R_{d} / d t$

$$
R_{d} \sim(I t)^{1 / 2}, \quad D_{\text {eff }} \sim I
$$

Equation (3) shows that the square-root growth is determined by currents (far above the limiting current) and $D_{\text {eff }}$ is linearly proportional to current, consistent with the experiments [Fig. 3(d)].

Additionally, for various constant voltage $(V), R_{d}$ can be fitted by a $1 / 2$ power-law scaling [Fig. 3(e)], and the extracted $D_{\text {eff }}$ increases with voltage [Fig. 3(f)]. Ion conservation at the thin shock interface implies $d R_{d} / d t \sim V / R_{d}$ (electric field for the radial geometry). Then square-root growth is obtained,

$$
R_{d} \sim(V t)^{1 / 2}, \quad D_{\text {eff }} \sim V .
$$

Indeed, the experimental $D_{\text {eff }}$ is linear with the voltages (far above the onset voltage) [Fig. 3(f)].

Remarkably, the EC vortices do not form chaotic random patterns and remain confined to a smooth envelope during DS propagation, as shown by the red smooth circular contours in Fig. 3(a). In hindsight, a DS radially moving away from the depletion region resembles time-reversed Laplacian growth [34], which leads to smooth shock profiles [27]. The stability of SC-driven DS is critical for continuous shock electrodialysis [21,29] and stable shock electrodeposition [11], so the observation of stable EC-driven DS may lead to related applications.

\section{MODEL FOR DS PROPAGATION}

Although 1/2 power-law scaling of shock propagation is predicted for SC-driven DS in a circular or wedge geometry [27], we employ our simple physical model to understand the similar dynamics of EC-driven DS. The shock velocity is proportional to the current density [27],

$$
\frac{d R_{d}}{d t} \sim j\left(R_{d}\right) \sim \frac{I}{2 \pi R_{d}} .
$$

For constant voltage $(V)$, assuming the voltage approximately dropped entirely in the depletion region with a constant $c_{d}$ independent on time, we find (Appendix D)

$$
I(t) \sim \frac{c_{d} V}{\ln \left(R_{d} / R_{1}\right)} .
$$




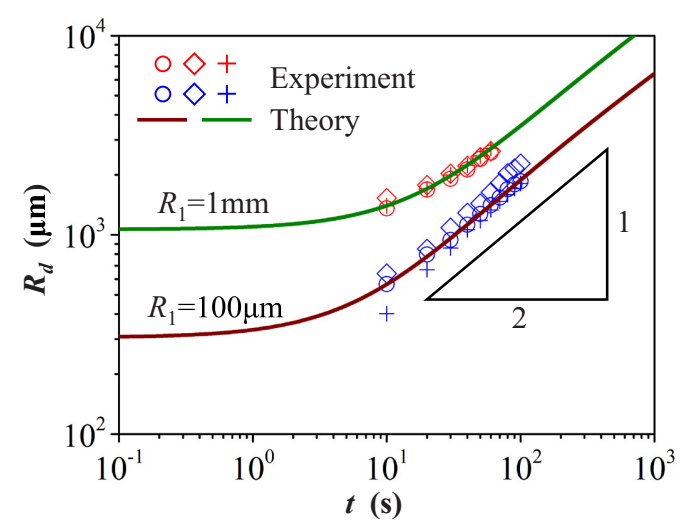

FIG. 4. Model for DS propagation. The experiment data under a constant voltage at $20 \mathrm{~V}$ are fitted well by Eq. (7). The power law of $1 / 2$ is recovered for a smaller radius $R_{1}=100 \mu \mathrm{m}(\chi=1 / 30)$, while deviation from $1 / 2$ power law occurs for a larger radius $R_{1}=1 \mathrm{~mm}(\chi=1 / 3)$. The lower bound of $R_{d}$ is limited by the extra liquids around the wire, and the upper bound of $R_{d}$ is $R_{2}(3 \mathrm{~mm})$. The experimental data were reproduced by three times.

Then $R_{d}(t)$ is obtained with a fitting parameter $\alpha$,

$$
\left(\frac{R_{d}}{R_{1}}\right)^{2}\left[\ln \left(\frac{R_{d}}{R_{1}}\right)^{2}-1\right]+1=\frac{\alpha c_{d} V}{R_{1}^{2}} t
$$

The above Eq. (7) is in excellent agreement with the experimental data (Fig. 4). For a smaller radius $R_{1}=100 \mu \mathrm{m}$, as shock propagates far away from the cathode $\left(R_{d} / R_{1}>1\right)$, the square-root growth holds $\left[R_{d} \sim(V t)^{1 / 2}\right]$, validating the aforementioned power-law analysis. But, for a larger radius $R_{1}=1 \mathrm{~mm}$, a length scale is set by $R_{1}$, and Eq. (7) including the logarithms term is more accurate than the power law. Despite the simplicity, the proposed model might have captured the main features of EC-driven DS.

\section{DISCUSSION}

Theoretically, three mechanisms-surface conduction, EOF, and EC-are responsible for the OLC. Experimentally, here the observed OLC, EC, and DS are unaffected by modified surface charges, thus ruling out the first two SC mechanisms, in which residual conductivity arises from excess counterions screening charged sidewalls. The simultaneous observation of vortices and concentration shock waves is quite challenging experimentally, and they had to be visualized in somewhat different systems to achieve accurate measurements (vortices in $1 \mathrm{mM}$ aqueous $\mathrm{CuSO}_{4}$ solution at $2 \mu \mathrm{A}$, and concentration shocks in $10 \mathrm{mM}$ compound solution at $20 \mu \mathrm{A}$ ).

For EOF and SC, scalings of the overlimiting conductance with reservoir salt concentration and channel thickness have been predicted [19] and confirmed experimentally [21,22]. It is beyond the scope of this paper to do the same for EC-driven OLC, but we note that our key model assumption, that $c_{d}$ is nearly constant during shock propagation, is consistent with previous studies of EC without geometrical confinement [13-15,19], and the model has a good agreement with DS propagation in experiments. Nevertheless, the simple model proposed here is only a first attempt to understand the interesting observations, and a more accurate and rigorous theory is required to quantitatively analyze the transition from the bulk to the depletions, likely resulting in the concentration discontinuity. Additionally, direct numerical simulations might be helpful to further thoroughly elucidate the underlying mechanism of EC-driven DS. 
Our results also hold for negative voltage bias $(\Phi<0)$, where vortices appear at the outer ring, while DS propagates inward at a higher current (Appendix C). It would also be interesting to test predictions of conformal invariance of ion transport in the absence of EC [35] by studying offcenter positions and diverse cross-sectional (elliptical or cloverlike) shapes [34] of the wire. The breakdown of conformal invariance in the transient problem also introduces flexibility to control DS stability [11].

Our observations of EC-driven DS are likely to also hold in other configurations, such as the imposed cross flow in shock electrodialysis [21,29] and microscale electrodialysis with vortices organized in the depleted region behind a fairly smooth DS [17]. This insight may provide guidance to achieve shock electrodialysis in bulk electrolytes without confinement by a charged porous medium, for example, in a simple electrodialysis-type stack with only one type of cation membrane, which may enable greater flow rates for continuous and scalable desalination due to the lower hydraulic resistance, albeit with the likely trade-off of lower desalination factor. Similar phenomena could also be exploited to control electrodeposition.

\section{CONCLUSION}

In conclusion, in a circular channel, a layer of bulk EC vortices appears in the horizontal plane to sustain OLC. The EC-driven depletion layer propagates radially as DS, and the propagation has a remarkable agreement with the proposed model. The EC-driven DS phenomenon may be exploited in new designs of shock electrodialysis for desalination and water purification.

\section{ACKNOWLEDGMENTS}

This work was supported by the National Young Thousand Talent Program in China and startup from Fudan University, the China Postdoctoral Science Foundation. S.M.R. was partially supported by DOE-PNNL under Grant No. 428977.

Z.G. and B.X. contributed equally to this work.

\section{APPENDIX A: OHMIC AND LIMITING CURRENT REGIMES WITHOUT EC}

When the EC instability (consequently the vortices) is absent, the residual conductivity vanished $\sigma_{E C}=0$ in the whole region $\left(\tilde{r}=r / R_{2}, \chi<\tilde{r}<1\right)$. Thus the $1 \mathrm{D}$ steady model equation (1) in the main text can be solved to the $1 \mathrm{D}$ quiscent solution of concentration polarization

$$
\begin{aligned}
& \tilde{c}=1-\frac{\tilde{I}}{4 \pi}\left(\ln \tilde{r}+\frac{1}{2}+\frac{\chi^{2} \ln \chi}{1-\chi^{2}}\right), \\
& \tilde{\phi}=\ln \tilde{c}+\text { const. }
\end{aligned}
$$

To obtain the solution (A1a), concentration conservation is imposed:

$$
\int_{\chi}^{1} \int_{0}^{2 \pi} \tilde{c} \tilde{r} d \tilde{r} d \theta=\int_{\chi}^{1} \int_{0}^{2 \pi} \tilde{r} d \tilde{r} d \theta=\pi\left(1-\chi^{2}\right) .
$$

Then from Eq. (A1b), there is a relation between the voltage $\tilde{V}=\tilde{\phi}(1)-\tilde{\phi}(\chi)$ across the circular channel and the current $\tilde{I}$ :

$$
1-\exp (\tilde{V})=\frac{\frac{\tilde{I}}{4 \pi} \ln \chi}{1-\frac{\tilde{I}}{4 \pi}\left(\frac{1}{2}+\frac{\chi^{2} \ln \chi}{1-\chi^{2}}\right)} .
$$

From Eqs. (A1), the concentration and potential profiles are different for the positive voltage bias (the cathode at the inner radius, $\tilde{r}=\chi, \tilde{I}<0$ ) and the negative voltage bias (cathode at the outer radius, $\tilde{r}=1, \tilde{I}>0$ ). For the positive voltage bias case, the $I-V$ relation (A3) is plotted out (solid line) in Fig. 1(b) in the main text. 
With a given geometry ratio $\chi$, the analytic solution of the limiting current under positive or negative voltage bias is obtained from Eq. (A1a) as the concentration approaches zero at cathode as below:

$$
\begin{aligned}
& \tilde{I}_{\text {pos }}=-8 \pi \frac{\left(1-\chi^{2}\right)}{1+2 \ln \chi-\chi^{2}} \quad[\tilde{c}(\chi)=0], \\
& \tilde{I}_{\text {neg }}=8 \pi \frac{\left(1-\chi^{2}\right)}{1+2 \chi^{2} \ln \chi-\chi^{2}} \quad[\tilde{c}(1)=0], \\
& \tilde{I}_{\text {bias }}=\frac{\tilde{I}_{\text {neg }}}{\tilde{I}_{\text {pos }}}=-\frac{1+2 \ln \chi-\chi^{2}}{1+2 \chi^{2} \ln \chi-\chi^{2}} .
\end{aligned}
$$

\section{APPENDIX B: OVERLIMITING CURRENT REGIME WITH EC}

The constant concentration $c_{d}$ in the depletion region can be matched with the concentration in the bulk region far away from the vortices by neglecting the transition layer between these two regions. The concentration distribution for the positive voltage bias case is obtained from Eq. (1) in the main text,

$$
\tilde{c}= \begin{cases}\tilde{c}_{d}, & \tilde{r} \leqslant \tilde{r}_{d}, \\ \frac{\tilde{I}}{4 \pi} \ln \left(\frac{\tilde{r}_{d}}{\tilde{r}}\right)+\tilde{c}_{d}, & \tilde{r}>\tilde{r}_{d} .\end{cases}
$$

The profile of electric field is also obtained from Eq. (1) in the main text,

$$
\tilde{E}=-\frac{d \tilde{\phi}}{d \tilde{r}}=\frac{\tilde{I}}{4 \pi \tilde{c} \tilde{r}} .
$$

For the given $\tilde{I}$ and $\tilde{c}_{d}, \tilde{r}_{d}$ of the depletion region is found by the concentration conservation (A2),

$$
\tilde{I}\left(1-\tilde{r}_{d}^{2}+2 \ln \tilde{r}_{d}\right)=8 \pi\left(1-\tilde{c}_{d}\right)\left(1-\chi^{2}\right) .
$$

And the total voltage is the sum of that in the bulk region $\ln \left[\tilde{c}(1) / \tilde{c}\left(\tilde{r}_{d}\right)\right]$ and the depletion region $\left[\sim \ln \left(\tilde{r}_{d} / \chi\right)=\ln \left(R_{d} / R_{1}\right)\right]$,

$$
\tilde{V}=\ln \left(1+\frac{\tilde{I}}{4 \pi \tilde{c}_{d}} \ln \tilde{r}_{d}\right)-\frac{\tilde{I}}{4 \pi \tilde{c}_{d}} \ln \left(\frac{R_{d}}{R_{1}}\right),
$$

which presents the $I-V$ relationship as well.

For the given $\tilde{c}_{d}$, by solving the coupled equations of (B3) and (B4), $I-V$ curves for $\tilde{c}_{d}=0.1$ and $\tilde{c}_{d}=0.01$ are also plotted in Fig. $1(\mathrm{~b})$ in the main text. For the given $\tilde{c}_{d}$ and $\tilde{V}$, the concentration distribution of Eq. (B1) and the profile of electric field of Eq. (B2) are shown in Figs. 1(c) and 1(d) in the main text.

\section{APPENDIX C: EXPERIMENTS RELATED WITH THE NEGATIVE VOLTAGE BIAS}

Based on Eq. (A4), the limiting current is dependent on the voltage bias. Indeed, the measured $I-V$ curves for $10 \mathrm{mM}$ aqueous $\mathrm{CuSO}_{4}$ solution show $I_{\text {pos }} \approx 0.4 \mu \mathrm{A}$ for the positive voltage bias and $I_{\text {neg }} \approx 2.25 \mu \mathrm{A}$ for the negative voltage bias [Figs. 5(a) and 5(b)]. Additionally, the limiting current is related with $\chi$, by varying $2 R_{1}=2,4$, and $5 \mathrm{~mm}(\chi=1 / 3,2 / 3,5 / 6)$; the obtained limiting currents at each given $\chi$ are normalized by their corresponding values at $\chi=1 / 30$. The normalized currents $(\tilde{I})$ are fitted well by Eq. (A4) [Fig. 5(c)], showing that ion transport in a circular channel can be controlled by the type of voltage bias and the diameter of the inner electrode. Under the negative voltage bias, the observed DS and vortices are presented in Fig. 6. 
(a)

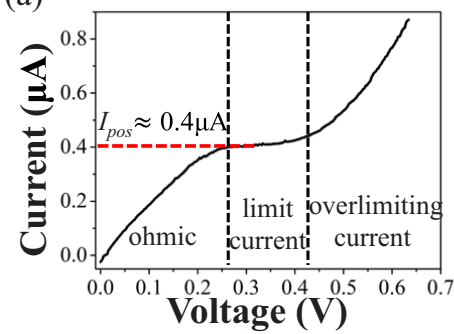

(b)

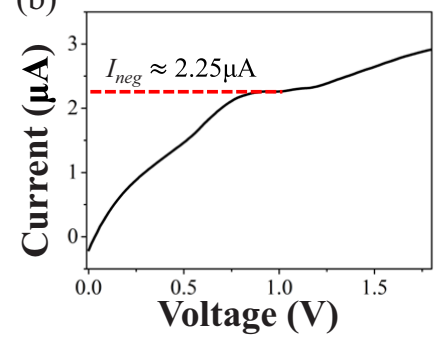

(c)

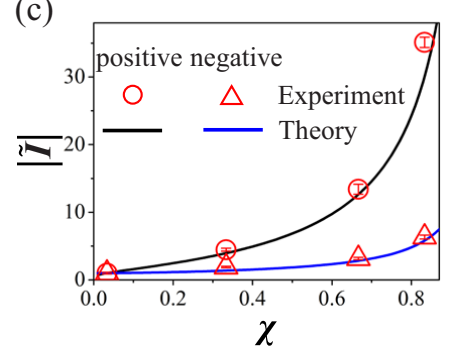

FIG. 5. Measured $I-V$ curves for $10 \mathrm{mM}$ aqueous $\mathrm{CuSO}_{4}$ solution showing (a) $I_{\text {pos }} \approx 0.4 \mu \mathrm{A}$ for the positive voltage bias and (b) $I_{\text {neg }} \approx 2.25 \mu \mathrm{A}$ for the negative voltage bias $(\chi=1 / 30)$. (c) $\tilde{I}$ agreeing well with Eq. (A4) and error bars for the standard deviations from four measurements.

\section{APPENDIX D: TIME-DEPENDENT CURRENT FOR CONSTANT VOLTAGE IN UNSTEADY STATE}

During the propagation of the DS, the Nernst-Planck equations in unsteady state are

$$
\begin{aligned}
& \frac{\partial c}{\partial t}=\frac{\partial}{\partial r}\left(r \frac{\partial c}{\partial r}+r c \frac{\partial \tilde{\phi}}{\partial r}+r \sigma_{E C} \frac{\partial \tilde{\phi}}{\partial r}\right), \\
& \frac{\partial c}{\partial t}=\frac{\partial}{\partial r}\left(r \frac{\partial c}{\partial r}-r c \frac{\partial \tilde{\phi}}{\partial r}+r \sigma_{E C} \frac{\partial \tilde{\phi}}{\partial r}\right) .
\end{aligned}
$$

In the depletion zone $\left\{\chi<r<r_{d}(t)\right\}$ with nearly constant concentration $c_{d}$, assuming this concentration $c_{d}$ is independent of time $t$, Eqs. (D1) above become

$$
2 c_{d} \frac{d \tilde{\phi}}{d r}=-\frac{c_{0} I \tilde{(t)}}{2 \pi r} .
$$

The assumption of the voltage approximately dropped entirely in this depletion region $\tilde{\phi}\left(r_{d}\right)-$ $\tilde{\phi}(\chi)=\tilde{V}$ yields

$$
\tilde{I}(t)=-\frac{4 \pi c_{d} \tilde{V}}{c_{0}} \frac{1}{\ln \left(r_{d} / \chi\right)}=-\frac{4 \pi c_{d} \tilde{V}}{c_{0}} \frac{1}{\ln \left(R_{d} / R_{1}\right)}
$$

(a)

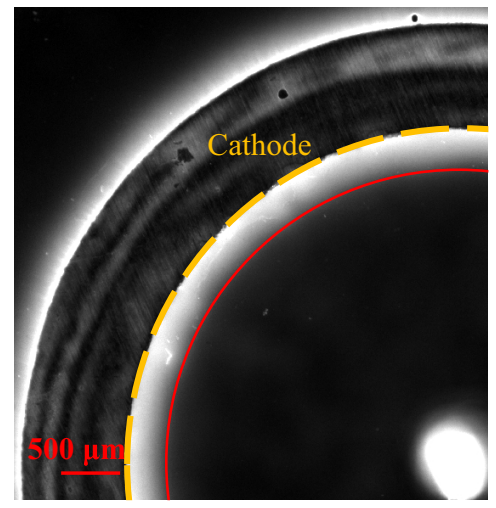

(b)

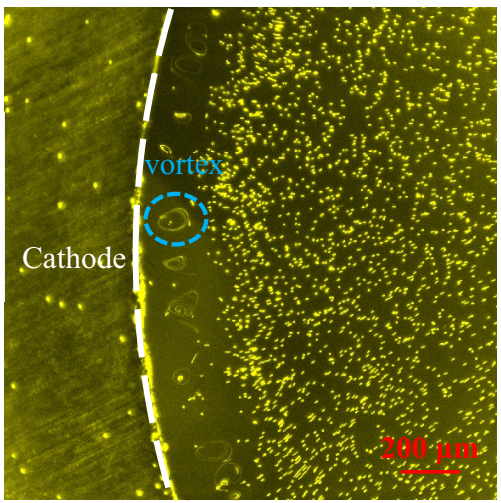

FIG. 6. Observed DS and vortices under the negative voltage bias $(\chi=1 / 30)$. (a) Snapshot of fluorescent signals at $60 \mathrm{~s}$ under an applied current at $10 \mu \mathrm{A}$; red line for the front position of DS. (b) An array of observed vortices near cathode side at $60 \mathrm{~s}$ under an applied current at $5 \mu \mathrm{A}$. 
[1] R. F. Probstein, Physicochemical Hydrodynamics (Wiley, New York, 2003).

[2] J. Newman and K. E. Thomas-Alyea, Electrochemical Systems (Wiley, New York, 2004).

[3] R. B. Schoch, J. Han, and P. Renaud, Transport phenomena in nanofluidics, Rev. Mod. Phys. 80, 839 (2008).

[4] A. A. Sonin and R. F. Probstein, A hydrodynamic theory of desalination by electrodialysis, Desalination 5, 293 (1968).

[5] Q. Pu, J. Yun, H. Temkin, and S. Liu, Ion-enrichment and ion-depletion effect of nanochannel structures, Nano Lett. 4, 1099 (2004).

[6] S. J. Kim, Y. Song, and J. Han, Nanofluidic concentration devices for biomolecules utilizing ion concentration polarization: theory, fabrication, and applications, Chem. Soc. Rev. 39, 912 (2010).

[7] S. Rubin, M. E. Suss, P. M. Biesheuvel, and M. Bercovici, Induced-Charge Capacitive Deionization: The Electrokinetic Response of a Porous Particle to an External Electric Field, Phys. Rev. Lett. 117, 234502 (2016).

[8] S. Porada, R. Zhao, A. Van Der Wal, V. Presser, and P. M. Biesheuvel, Review on the science and technology of water desalination by capacitive deionization, Prog. Mater. Sci. 58, 1388 (2013).

[9] V. V. Nikonenko, A. V. Kovalenko, M. K. Urtenov, N. D. Pismenskaya, J. Han, P. Sistat, and G. Pourcelly, Desalination at overlimiting currents: State-of-the-art and perspectives, Desalination 342, 85 (2014).

[10] J.-H. Han, E. Khoo, P. Bai, and M. Z. Bazant, Over-limiting current and control of dendritic growth by surface conduction in nanopores, Sci. Rep. 4, 7056 (2014).

[11] J.-H. Han, M. Wang, P. Bai, F. R. Brushett, and M. Z. Bazant, Dendrite suppression by shock electrodeposition in charged porous media, Sci. Rep. 6, 28054 (2016).

[12] M. B. Andersen, M. Van Soestbergen, A. Mani, H. Bruus, P. M. Biesheuvel, and M. Z. Bazant, CurrentInduced Membrane Discharge, Phys. Rev. Lett. 109, 108301 (2012).

[13] I. Rubinstein and B. Zaltzman, Electro-osmotically induced convection at a permselective membrane, Phys. Rev. E 62, 2238 (2000).

[14] B. Zaltzman and I. Rubinstein, Electro-osmotic slip and electroconvective instability, J. Fluid Mech. 579, 173 (2007).

[15] S. M. Rubinstein, G. Manukyan, A. D. Staicu, I. Rubinstein, B. Zaltzman, R. G. H. Lammertink, F. G. Mugele, and M. Wessling, Direct Observation of a Nonequilibrium Electro-Osmotic Instability, Phys. Rev. Lett. 101, 236101 (2008).

[16] G. Yossifon and H.-C. Chang, Selection of Nonequilibrium Overlimiting Currents: Universal Depletion Layer Formation Dynamics and Vortex Instability, Phys. Rev. Lett. 101, 254501 (2008).

[17] R. Kwak, G. Guan, W. K. Peng, and J. Han, Microscale electrodialysis: Concentration profiling and vortex visualization, Desalination 308, 138 (2013).

[18] I. Rubinstein and B. Zaltzman, Equilibrium Electroconvective Instability, Phys. Rev. Lett. 114, 114502 (2015).

[19] E. V. Dydek, B. Zaltzman, I. Rubinstein, D. Deng, A. Mani, and M. Z. Bazant, Overlimiting Current in a Microchannel, Phys. Rev. Lett. 107, 118301 (2011).

[20] E. V. Dydek and M. Z. Bazant, Nonlinear dynamics of ion concentration polarization in porous media: The leaky membrane model, AIChE J. 59, 3539 (2013).

[21] D. Deng, E. V. Dydek, J. Han, S. Schlumpberger, A. Mani, B. Zaltzman, and M. Z. Bazant, Overlimiting current and shock electrodialysis in porous media, Langmuir 29, 16167 (2013).

[22] S. Nam, I. Cho, J. Heo, G. Lim, M. Z. Bazant, D. J. Moon, G. Y. Sung, and S. J. Kim, Experimental Verification of Overlimiting Current by Surface Conduction and Electro-Osmotic Flow in Microchannels, Phys. Rev. Lett. 114, 114501 (2015).

[23] E. Khoo and M. Z. Bazant, Theory of voltammetry in charged porous media, J. Electroanal. Chem. 811, 105 (2018).

[24] A. Mani, T. A. Zangle, and J. G. Santiago, On the propagation of concentration polarization from microchannel-nanochannel interfaces part i: analytical model and characteristic analysis, Langmuir 25, 3898 (2009). 
[25] T. A. Zangle, A. Mani, and J. G. Santiago, On the propagation of concentration polarization from microchannel-nanochannel interfaces part ii: numerical and experimental study, Langmuir 25, 3909 (2009).

[26] T. A. Zangle, A. Mani, and J. G. Santiago, Theory and experiments of concentration polarization and ion focusing at microchannel and nanochannel interfaces, Chem. Soc. Rev. 39, 1014 (2010).

[27] A. Mani and M. Z. Bazant, Deionization shocks in microstructures, Phys. Rev. E 84, 061504 (2011).

[28] D. Deng, W. Aouad, W. A. Braff, S. Schlumpberger, M. Suss, and M. Z. Bazant, Water purification by shock electrodialysis: Deionization, filtration, separation, and disinfection, Desalination 357, 77 (2015).

[29] S. Schlumpberger, N. B. Lu, M. E. Suss, and M. Z. Bazant, Scalable and continuous water deionization by shock electrodialysis, Environ. Sci. Technol. Lett. 2, 367 (2015).

[30] A. Yaroshchuk, Over-limiting currents and deionization "shocks" in current-induced polarization: Localequilibrium analysis, Adv. Colloid Interface Sci. 183, 68 (2012).

[31] J. De Valenca, R. M. Wagterveld, R. G. H. Lammertink, and P. A. Tsai, Dynamics of microvortices induced by ion concentration polarization, Phys. Rev. E 92, 031003(R) (2015).

[32] See Supplemental Material at http://link.aps.org/supplemental/10.1103/PhysRevFluids.4.113701 for Videos 1 and 2.

[33] E. Karatay, M. B. Andersen, M. Wessling, and A. Mani, Coupling Between Buoyancy Forces and Electroconvective Instability Near Ion-Selective Surfaces, Phys. Rev. Lett. 116, 194501 (2016).

[34] M. Z. Bazant, Interfacial dynamics in transport-limited dissolution, Phys. Rev. E 73, 060601(R) (2006).

[35] M. Z. Bazant, Conformal mapping of some non-harmonic functions in transport theory, Proc. R. Soc. London A 460, 1433 (2004). 